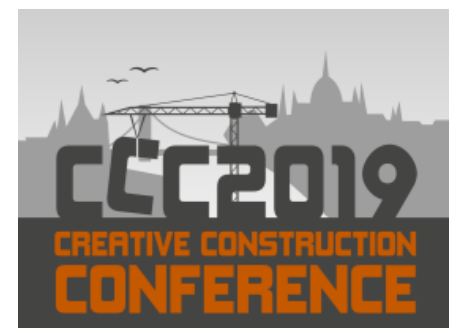

Available online at 2019.creative-construction-conference.com/proceedings/

CCC 2019

Proceedings of the Creative Construction Conference (2019) 075

Edited by: Miroslaw J. Skibniewski \& Miklos Hajdu

https://doi.org/10.3311/CCC2019-075

Creative Construction Conference 2019, CCC 2019, 29 June - 2 July 2019, Budapest, Hungary

\title{
Applied decision-making framework for maintenance scheduling in bridge management
}

\author{
Niloofar Montazeri ${ }^{\mathrm{a}, *}$, Ali Touran ${ }^{\mathrm{b}}$ \\ ${ }^{a}$ Keville Enterprises, Inc, 145 Tremont St, Boston, MA 02111 \\ ${ }^{b}$ Northeastern University, 360 Huntington Ave, Boston, MA 02115
}

\begin{abstract}
The deterioration of bridge structures in the United States is a national multibillion-dollar problem. Recent studies and reports show that almost $10 \%$ of these bridges need to be repaired or replaced. Therefore, facilitating decision-making for optimal bridge maintenance scheduling is essential for the management of networks of these aging structures where lack of preventative and proactive strategies cause significant consequences. In order to achieve such schedule, analytical methods are required to optimize conflicting factors over the life-cycle of the bridge while considering (i) structural performance and (ii) maintenance cost.

An advanced reliability-based decision-making framework for maintenance scheduling of deteriorating bridges is proposed. The outcome is a decision-making framework regarding repair and replacement of the structure over its life-cycle in terms of schedule and type of action to-be-performed on selected components considering system requirements, structure specification, as well as available budget. To implement the proposed framework, and in order to evaluate the performance of the developed methodology, a numerical experiment is developed. The results obtained by applying the methodology on an existing bridge are analyzed and the efficiency and advantages of the procedure are demonstrated.
\end{abstract}

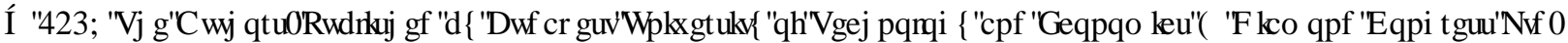

Peer-review under responsibility of the scientific committee of the Creative Construction Conference 2019.

Keywords: bridge management; reliability-based maintenance scheduling; decision-making framework; multi-objective optimization

\section{Introduction}

In recent years, in order to facilitate the efficient decision-making for scheduling maintenance for bridge systems, many performance models have been proposed, and various optimization methods have been introduced for obtaining the optimal maintenance plans using life-cycle performance and cost-efficiency in deteriorating structures. A class of these methods uses annual reliability index as a performance indicator where multi-objective optimization has been applied to reach the solutions. Most of these approaches deal with limited maintenance actions such as preventative measures $[1,2]$. On the other hand, probabilistic models only deal with the uncertainties related to the indicators regarding the performance of the system, not the maintenance cost. Accordingly, in the previous methods, a comprehensive category for maintenance actions has not been considered, cost models have been assumed deterministic, and also optimization approaches have been bounded to a set of feasible solutions - not an optimal solution. In bridge management, optimal maintenance plans play a crucial role in the strategic decision-making. These 
Niloofar Montazeri and Ali Touran/ Proceedings of the Creative Construction Conference (2019) 075 https://doi.org/10.3311/CCC2019-075

plans have to address a number of conflicting factors and criteria. To achieve optimal or close to optimal plans, advanced analytical methods are necessary to optimize maintenance cost and increase the performance level of bridges.

In this paper, we focus on development of a maintenance scheduling method to enrich previous works and increase the efficiency of the maintenance decision-making process by overcoming mentioned limitations. The objective is to propose a decision-making framework for scheduling the maintenance of bridge superstructure as a multi-component system using multi-objective optimization to maximize structural performance - annual reliability index - and minimize life-cycle total maintenance cost to suggest a sequence of maintenance actions performed on selected components of the bridge. The approach is implemented on an existing deteriorating bridge superstructure. Main features of the approaches proposed in this paper include (1) considering a spectrum of maintenance actions including prevention, different levels for repair, and replacement, (2) performing optimization over probabilistic maintenance cost model, and (3) achieving ultimate optimal solution by solving optimization problem for Pareto frontier associated with optimal maintenance schedules of the structural system.

Infrastructure systems are subject to failure caused by several phenomena such as resistance reduction and increase of imposed loads, which lead to reduction of structural safety. When failure happens, repair or replace action is necessary. In order to keep the structure reliable, optimal maintenance strategy is required considering constraints, uncertainties, and economic factors during the life-cycle of the system. This needs an effective decision-making procedure and framework to reach optimality in planning. Methods and techniques for the determination of life-cycle maintenance strategies and plans have been extensively discussed in recent years and many approaches have been proposed. Advanced methods apply economic, environmental, structural and safety requirements to optimize time-dependent structural performance considering uncertainties [3].

Deterioration of existing structures due to increasing loads over time, environmental causes, and material properties have been studied over decades. If proper maintenance plan is not considered, this phenomenon leads to loss of reliability and serviceability of the structure as well as structural failure. In addition, obtaining economic plan and reducing unnecessary costs are required. Therefore, applying a cost-effective maintenance plan to avoid deterioration and damage is a significant problem in infrastructure systems, such as highway bridges.

Optimal maintenance scheduling of structures and infrastructures requires the development of methodologies for generating cost-effective optimal strategies for bridge maintenance scheduling including time and types of actions. Maintenance actions are categorized as (1) preventive, which is slowing down the structural deterioration, (2) repairing, and (3) replacing a failed or damaged component $[1,3,4]$. Among the techniques used in decision-making, multiobjective optimization can provide optimal solutions considering all complexities and limitations $[3,4,5]$.

Uncertainties associated with load, environment, and material make the accurate prediction and planning complicated. Therefore, structural performance such as reliability should be predicted in terms of a probabilistic metric. In addition, uncertainties associated with time and cost make the exact prediction and planning impossible. Considering all of these uncertainties, the optimal maintenance scheduling for bridge structures should be reliability-based. In recent years, advancements in structural reliability theory and life-cycle stochastic optimization, have developed new tools and techniques for infrastructure systems analysis under above-mentioned uncertainties [1, 3, 4]. Therefore an advanced reliability-based maintenance optimization methodology for deteriorating bridges to find the optimum maintenance action sets including schedule and type of action is required. To generate such methodology for optimum maintenance scheduling procedure and to propose an applied decision-making framework associated with the life-cycle of the bridge, a multi-objective optimization considering total maintenance cost and system structural performance reliability index - is necessary. 
Niloofar Montazeri and Ali Touran/ Proceedings of the Creative Construction Conference (2019) 075 https://doi.org/10.3311/CCC2019-075

As an advanced solution, maintenance costs and structural performance index are projected by exhaustive search and scheduling sets obtained by Pareto Frontier are applied in a multi-objective optimization method to report optimal maintenance schedules. This paper proposes an advanced methodology for determining optimal bridge maintenance scheduling based on probabilistic total maintenance cost and minimum annual system reliability index as structural performance metric through a decision-making framework. The approach is fully developed and programmed using the MATLAB ${ }^{\circledR} \mathrm{R} 2011 \mathrm{~b}$ programming environment.

\section{Methodology}

Analyzing performance of structural systems of bridges is necessary for optimizing maintenance cost including repair and replacement. As a continuation of previous related works, which were focused on replacement strategies, this paper pursues an approach for optimizing repair and replacement in bridge maintenance. The aim of this paper is to propose a multi-objective method to optimize the cost for bridge repair and replacement using probabilistic performance indicator - system reliability index. This procedure is introduced in three phases. The first phase preliminary - includes preliminaries for this methodology including data fitting, structural modeling, optimization parameter definitions, reliability calculation, and setting the policies. In the second phase - implementation - steps for repair and replace procedure are proposed. Multi-objective optimization is discussed in the third phase - optimization. We discuss these phases next.

\subsection{Preliminary Phase}

The input for the proposed procedure is the initial data including the bridge structural reliability model and reliability index for each independent bridge component available in a certain life-cycle. Reliability indices for each component over the bridge life-cycle are used for polynomial fitting in order to reach a complete set of reliability indices data for all years in the life-cycle.

By making appropriate series, parallel and combined system assumptions, we model the bridge structural system reliability. The reliability of a bridge structural system is evaluated by considering the system failure as series-parallel combination of the component failures. The primary assumption in the bridge superstructure reliability modeling is that the system failure is considered to occur when either deck fails or any two adjacent girders fail [6]. For instance, collapse of deck causes the system failure. This assumption is limited to the linear interaction between the components [6].

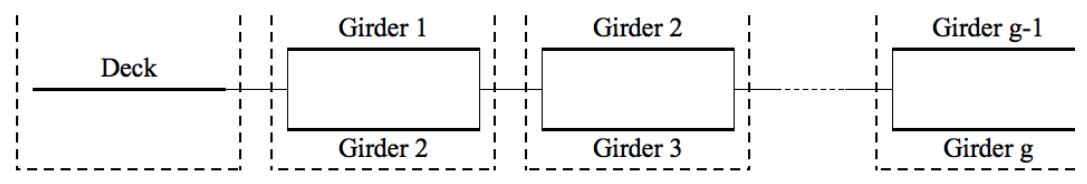

Fig. 1. System Reliability Model for the Bridge Superstructure

The system reliability model for the bridge superstructure is constructed by $n$ sub-systems as illustrated in Figure 1 . Each block, numbered 1 to $n$, represents a sub-system in this series structural reliability model. We show the deck with " $D$ " and girders with " $G$ ". Sub-system 1 represents $D$, where sub-systems 2 to $n$ are parallel model of two girders each. Structural reliability calculations for all sub-systems over the life-cycle of the superstructure are:

$$
R_{1}=R_{D} \quad \text { and } \quad R_{i}=1-\left(1-R_{G, i-1}\right)\left(1-R_{G, i}\right) \quad i=2 \ldots g
$$

Therefore, sub-system reliability calculations are obtained as following: 
Niloofar Montazeri and Ali Touran/ Proceedings of the Creative Construction Conference (2019) 075 https://doi.org/10.3311/CCC2019-075

$$
\beta_{j}=-\Phi^{-1}\left(1-R_{j}\right) \quad j=1 \ldots m
$$

System Reliability $\left(R_{\text {sys }}\right)$ is defined as the minimum of the sub-system reliabilities in a series structural reliability model [7]. Similarly, System Reliability Index $\left(\beta_{\text {sys }}\right)$ is the minimum of the sub-system reliability indices. In the series structural reliability model, the system reliability index over life-cycle are defined as:

$$
\beta_{\text {sys }}=\min \left\{\beta_{i}\right\} ; \quad i=1 \ldots m
$$

where $R_{i}$ and $\beta_{i}$ are reliability and reliability index for $i$ 'th sub-system respectively and $m$ is the number of subsystems.

The defined number of condition states in the proposed approach is 1 to 5 that comprising of good, fair, poor, very poor, and severe respectively. According to [8], condition state for bridge components is estimated using a piecewise linear function of time as following by step - floor - function:

$$
\begin{gathered}
C=\lfloor 1+\alpha t\rfloor \\
C^{*}= \begin{cases}C, & C \leq 5 \\
5, & C>5\end{cases}
\end{gathered}
$$

The constant piece of this function represents the maximum possible value for condition state after a certain deterioration condition, equal to 5 . We set an action policy and define 5 different action types pertaining to each condition state including: a preventive action, three level of repair action, and a replace action. Examples of this setting are DOTs in the states of Minnesota, Colorado, and Florida. This general action policy is presented as following:

$$
\left\{\begin{array}{c}
C S=1 ; \text { Preventive action } \\
2 \leq C S \leq 4 ; \text { Repair action } \\
5 \leq C S ; \text { Replace action }
\end{array}\right.
$$

If the action type recognized as repair action, when condition state is between 2 and 4 , the repair policy is applied as:

$$
C S=x ; \text { Repair to } C S=x-1
$$

To determine type of repair/replace actions, we calculate boundaries over condition stated for each component.

We use exhaustive (brute-force) enumeration algorithm to generate all possible repair/replace (action) time sets in a defined life-cycle. Each subset is called a scenario. Therefore, for a bridge life-cycle of $T_{L C}$ years, number of maintenance actions is considered as $N_{r}$. Minimum time between two consecutive maintenance actions is defined as $T_{\Delta}$, and the efficient life-cycle for applying any maintenance action is $T_{E F}$ years.

$$
\left\{\begin{array}{l}
t_{j+1}-t_{j} \geq T_{\Delta} \text { years } \\
t_{N_{r}} \leq T_{E F} \text { years }
\end{array} \quad j=1 \ldots\left(N_{r}-1\right)\right.
$$

Where $t_{N_{r}}$ is the maintenance time. For example, we can set bridge life-cycle $T_{L C}=75$, number of maintenance actions $N_{r}=3$, minimum time between two consecutive maintenance actions $T_{\Delta}=6$, and the efficient life-cycle for applying any maintenance action $T_{E F}=60$. The outcome of the exhaustive enumeration model is a $p$-by- $q$ matrix of scenarios times called $T C$, where $q=N_{r}$, and $p$ is the number of all possible scenarios.

\subsection{Implementation Phase}

The objective of this process is to apply brute-force search over enumerated scenarios by determining to-berepaired/replaced component at each selected time point in a scenario, applying appropriate repair/replace action, and updating components, sub-systems, and system reliability indices and condition states [9]. Using action policy and repair policy, we define target condition state corresponding to each policy. We use this information for updating the 
Niloofar Montazeri and Ali Touran/ Proceedings of the Creative Construction Conference (2019) 075 https://doi.org/10.3311/CCC2019-075

component reliability index and consequently re-calculating sub-systems and system reliability index for the remaining life of the superstructure. We need to recognize the component that requires repair or replace action. Considering enumeration search, we analyze each scenario one-by-one and report to-be-repaired/replaced component in each action time. To obtain this objective, we introduce three models based on different approaches for determining to-berepaired/replaced component as following:

Model A: Component-based Approach

In this model, we define a sub-procedure, which is able to report an action binary indicator regarding each of $n$ components at each action time where:

$$
\left\{\begin{array}{l}
\delta_{i}=1 ; \text { action required } \\
\delta_{i}=0 ; \text { no action required } \quad i=1 \ldots n
\end{array}\right.
$$

At each action time, the outcome vector of this sub-procedure contains $n-1$ component indicators equal to 0 and only one component indicator equal to $1 ; \exists ! i \leq n\left(\delta_{i}=1\right)$. Component with the indicator of 1 is considered as to-berepaired/replaced component. The component corresponding to $\delta_{i}=1$ has the minimum value of reliability index among all components at a time.

\section{Model B: Sub-system-based Approach}

In this model, in order to report an action binary indicator regarding each component at each action time, another subprocedure is defined to assign an indicator to each of $m$ sub-systems as:

$$
\left\{\begin{array}{l}
\theta_{i}=1 ; \text { action required } \\
\theta_{i}=0 ; \text { no action required } \quad i=1 \ldots m
\end{array}\right.
$$

and also to each of $n$ components as:

$$
\left\{\begin{array}{l}
\delta_{j}=1 ; \text { action required } \\
\delta_{j}=0 ; \text { no action required } \quad j=1 \ldots n
\end{array}\right.
$$

At each action time, the outcome vector of this sub-procedure contains $m-1$ sub-system indicators equal to 0 and only one sub-system indicator equal to $1 ; \exists ! i \leq m\left(\theta_{i}=1\right)$, as well as $n-1$ component indicators equal to 0 and only one component indicator equal to $1, \exists ! j \leq n\left(\delta_{j}=1\right)$. Sub-system with the indicator of 1 is subject to action and component with the indicator of 1 is considered as to-be-repaired/replaced component. The approach to reach this outcome is to select the sub-system corresponding to $\theta_{i}=1$ with the minimum value of sub-system reliability index among all other sub-systems at a time. As a result, to-be-repaired/replaced component is determined as the component with the minimum value of reliability index among all components in the selected sub-system.

\section{Model C: Hybrid Approach}

Two different models A and B use component-based and sub-system-based approaches respectively. The procedure in the approach of Model C starts with a comparison of outcomes from Model A and Model B at a time [9]. If those outcomes are similar indicating same component as the to-be-repaired/replaced, then the to-be-repaired/replaced component will be reported as the selected one in the Models A and B. If those outcomes report different components as the to-be-repaired/replaced, then Model $\mathrm{C}$ is activated. In this situation, a lower limit reliability index for a component is defined as $\beta^{l}$. Let's call the selected to-be-repaired/replaced components by Model $\mathrm{A}$ as $\Delta^{A}$ and by Model $\mathrm{B}$ as $\Delta^{B}$. If the selected to-be-repaired/replaced component by Model $\mathrm{C}$ is $\Delta^{C}$, we define the procedure for Model $\mathrm{C}$ as following:

$$
\text { If } \Delta^{A} \neq \Delta^{B} \text { then } \Delta^{C}=\left\{\begin{array}{l}
\Delta^{A} ; \beta_{\Delta^{A}} \leq \beta^{l} \\
\Delta^{B} ; \beta_{\Delta^{A}}>\beta^{l}
\end{array}\right.
$$


Niloofar Montazeri and Ali Touran/ Proceedings of the Creative Construction Conference (2019) 075 https://doi.org/10.3311/CCC2019-075

Model C allows us to use the results of Model B considering the critical condition of the suggested to-berepaired/replaced component by Model A. In other words, if the reliability index of the selected components by Model A is not less that defined lower limit, Model C prefers to report the selected component by Model B. The advantages of the hybrid model (Model C) are discussed in next section along with results and analysis. For each scenario obtained by exhaustive (brute-force) enumeration model, we use the outcome Model A, B, or C in order to (1) apply required repair/replace action, (2) update components, sub-systems, and system reliability indices, and (3) find Minimum System Reliability Index $\left(\beta_{s y s}^{m}\right)$ as the minimum value of the system reliability indices in the life-cycle of each scenario.

At each scenario, we calculate cumulative amount of future values for cost of actions for defined $\mathrm{N}_{\mathrm{r}}$ actions to obtain total cost of action for the scenario. Using obtained action binary indicator $\delta$ for each component at each action time $t$, we can determine the cost of action $C^{a}$ at each action time by using action binary indicators as:

$$
C^{a}=\delta_{1} C_{1}^{*}+\delta_{2} C_{2}^{*}+\cdots+\delta_{n} C_{n}^{*}=\sum_{k=1}^{n} \delta_{k} C_{k}^{*}
$$

where generally $C^{a}$ represent the cost of an action at each time, and $C_{j}^{a}$ indicate the cost of $j$-th action in a scenario. The total cost of action for $i$-th scenario $C T_{i}$ is achieved as following:

$$
C T_{i}=\sum_{j=1}^{N_{r}} \sum_{k=1}^{n} \frac{\delta_{k} C_{k}(1+i)^{t_{j}}}{(1+r)^{t_{j}}}
$$

In the next phase, we convert multi-objective optimization for cost-reliability of superstructure.

\subsection{Optimization Phase}

We use "no-preference scalarized weighted metric method" for multi-objective optimization in order to find optimal solution among the Pareto Frontier obtained in the previous step. This multi-objective optimization method does not require any preference information to be explicitly articulated $[10,11]$. The scalarized problem is formulated as

$$
\min \|f(x)-z\|_{2} \quad \text { s.t. } x \in X
$$

where $z$ represents ideal value preferred by the decision maker. This value for total maintenance cost $(t m c)$ is the minimum value in the vector $C T$ as:

$$
z_{C T}=\min \left(C T_{i}\right) ; \quad i=1 \ldots N_{\text {par }}
$$

where $C T$ (CostTotal) is a vector containing $C T$ s for all possible scenarios.

The ideal for minimum annual system reliability index (masr) is the maximum value in the vector $B M$ as:

$$
z_{B M}=\max \left(B M_{i}\right) ; i=1 \ldots N_{\text {par }}
$$

where $B M$ (BetaMin) including the minimum value of system reliability index corresponding to each scenario, and $N_{\text {par }}$ is the number of scenarios on the Pareto frontier. Therefore, our problem is modeled as following:

$$
\varepsilon=\arg \min \mathcal{H}(B M, C T):=\left[\left(B M_{i}-z_{B M}\right)^{2}+\left(C T_{i}-z_{C T}\right)^{2}\right]^{1 / 2}
$$

The scenario corresponding to the argument $\varepsilon$ is the optimal solution over Pareto frontier. By applying above procedure for multi-objective optimization of bridge repair and replace, we obtain optimal decision-making regarding the cost for bridge repair and replacement of the structure during the life-cycle. We present implementation of this process along with a numerical example next. 
Niloofar Montazeri and Ali Touran/ Proceedings of the Creative Construction Conference (2019) 075 https://doi.org/10.3311/CCC2019-075

\section{Results and Analysis}

To implement the proposed framework for reliability-based maintenance scheduling approach, and in order to evaluate the performance of introduced methodology, a numerical experiment is developed. This example shows the application of the methodology for any given bridge when the structural specifications and the life-cycle information are available.

The methodology is applied on bridge E-16-LY, Colorado (US Highway 36) built in 1985, in order to analyze the results. This bridge was introduced in [5]. This bridge superstructure contains three types of components; deck, interior girder, and exterior girder. This bridge has 3 spans. We implement the proposed procedure on the middle span of the superstructure of this bridge. The proposed procedure can be used with various bridges with different structures. In order to utilize this procedure for an existing superstructure, we need structural model configuration information and reliability indices of the bridge components (deck and girders) over the life-cycle as well as cost information of different type of maintenance actions for each component (replace and various levels of repair). We present results of applying the methodology for three models $\mathrm{A}, \mathrm{B}$, and C.
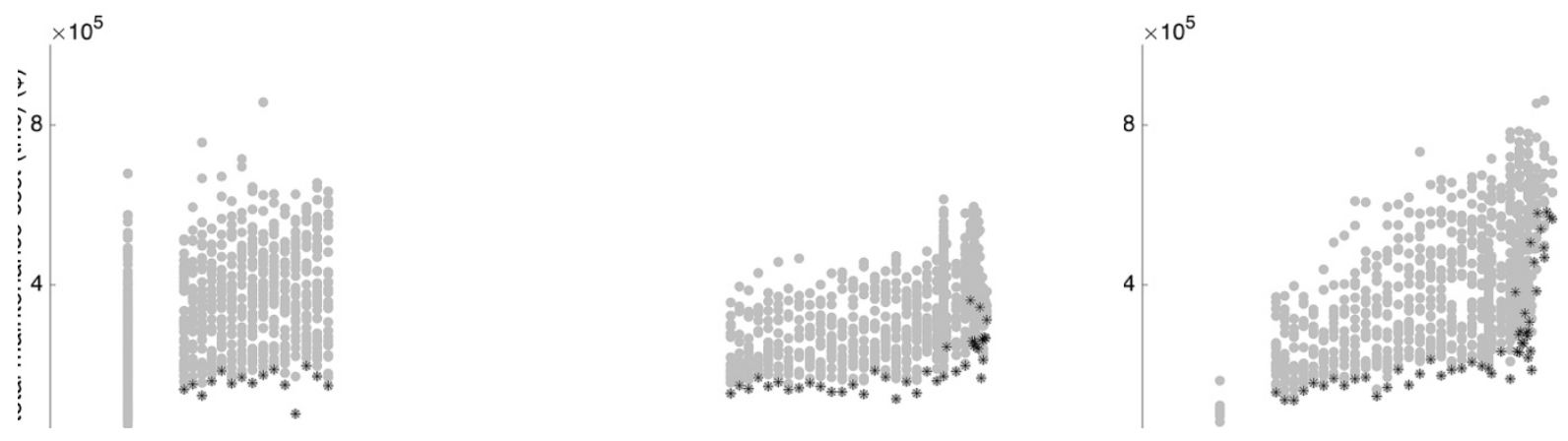

Fig. 2. Pareto Frontier (Black Asterisk) Bridge E-16-LY, Models A (left), B (middle), and C (right), $N_{r}=2$

Figure 2 represents the obtained Pareto Frontiers of Models A, B, and C with two maintenance actions (repair/replace) for bridge E-16-LY, respectively. Comparison of the results of Model C to Models A and B shows that Model C (Hybrid Model) increases the efficiency of the proposed methodology which is (i) decreasing the total maintenance cost, or (ii) reaching greater minimum annual system reliability.
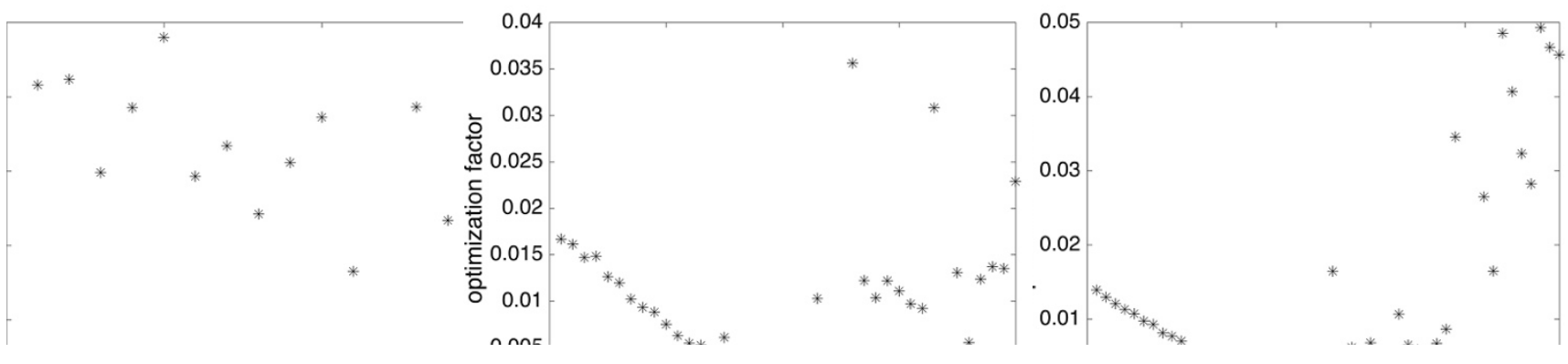

Fig. 3. Optimal Solution (Orange Asterisk) Bridge E-16-LY, Models A (Top), B (Middle), and C (Bottom), $N_{r}=2$ 
Niloofar Montazeri and Ali Touran/ Proceedings of the Creative Construction Conference (2019) 075 https://doi.org/10.3311/CCC2019-075

Setting two required maintenance actions in the life-cycle $\left(N_{r}=2\right)$ and applying the procedure, the obtained Pareto frontier including selected candidate scenarios for each model is used as an input for the weighted single-objective optimization (sum of squared error) to report the best solution. By implementing "no-preference scalarized weighted metric method" for multi-objective optimization over each three obtained Pareto frontier sets for any subject bridge, we find the optimal solution among these selected scenarios. As a result of multi-objective optimization for minimum annual system reliability (performance indicator) and total maintenance cost, the optimal scenario is a scenario in the life-cycle of the subject bridge. This scenario suggests optimal maintenance actions. The results present types of actions contain to-be-repaired/replace component and the level of repair/replace, and also times of these desired actions. Figures 3 depict the optimal solution over the Pareto Frontier.

The optimal solution over Pareto Frontier (Orange Asterisk in Figure 3) in Model A is a scenario with the performance indicator value equal to 1.9264 and the maintenance cost equal to $\$ 77,713$ (Scenario \#12). These values for the optimal solution in Model B (Scenario \#19) are 2.5113 and \$129,316, where Model C (Hybrid Model) reaches the value 2.8920 for performance indicator and \$164,607 for maintenance cost in its optimal solution (Scenario \#25). Therefore the obtained optimal solution by Model $\mathrm{C}$ is more reliable and this model could reach higher values for masr on the Pareto Frontier.

By applying multi-objective optimization over Pareto Frontier as a framework for partially evaluating feasible solutions, the optimal solution is obtained which satisfy one or more objectives, but not all. This optimal solution may change by considering boundaries and constraints by experts as "personal preferences of the decision maker". Under certain circumstances, cut-offs may be considered for performance indicator (reliability index) or maintenance cost, or both. Definitions of minimum annual system reliability (masr) by Departments of Transportation (DOTs) are different.

Experts may put a limit on "masr" and ignore an area of the Pareto Frontier by performance indicator cut-off, which eliminates some scenarios, and make the optimization more accurate, to the point. Also, if the maintenance budget is limited, proper cost cut-off may apply on the Pareto Frontier, eliminating some scenarios. Figure 4 depicts this concept for an example of minimum annual system reliability more than 2 and total maintenance cost (budget) less than $\$ 300,000$ for the Model C Pareto Frontier of bridge E-16-LY.

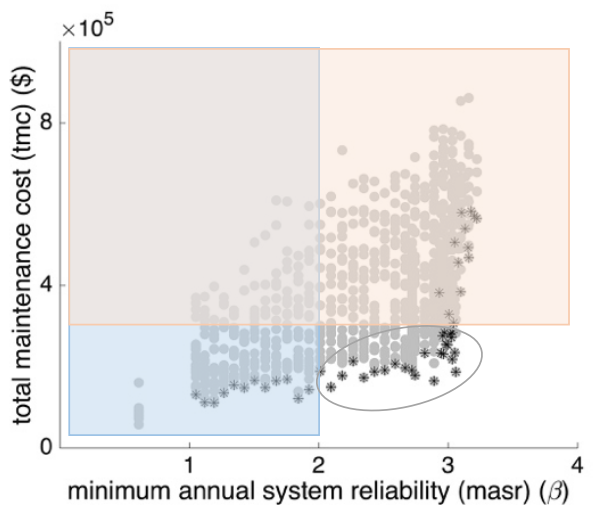

Fig. 4. Adjusted Pareto Frontier (Black Asterisk in Green Oval) Bridge E-16-LY, Model C, $N_{r}=2$, masr $>2$

(Blue Area Eliminated), tmc $<\$ 300,000$ (Red Area Eliminated) 
Niloofar Montazeri and Ali Touran/ Proceedings of the Creative Construction Conference (2019) 075 https://doi.org/10.3311/CCC2019-075

\section{Conclusion}

Deterioration of bridge structures is a national issue in the United States. Facilitating decision-making for optimal bridge maintenance scheduling is crucial and plays a significant role in bridge management. Optimal maintenance schedule is required to keep the structure performing in a reliable manner considering constraints, uncertainties, and economic factors during the life-cycle of the system. To achieve this, an advanced and comprehensive analytical method is required to optimize maintenance cost and maintain or improve the structural performance of bridges.

Previous methods (i) lack a comprehensive category for the maintenance actions, (ii) assume deterministic cost models, (iii) focus on deck and girder replacement strategies only, and (iv) result in optimization approaches bounded to a set of feasible solutions - not an optimal solution. To advance previous works and to overcome mentioned limitations in maintenance decision-making process, a reliability-based multi-objective optimization methodology for bridges is proposed, where the condition states, the probabilistic annual maintenance cost and the system structural performance indicator - reliability index - are projected by exhaustive search and scheduling sets are obtained from Pareto Frontier.

The inputs to the procedure are (i) structural model configuration (reliability model) and structural specifications of the bridge components (reliability indices) including deck and girders over the life-cycle, (ii) probabilistic cost of different type of maintenance actions for each component including replace and various levels of repair, (iii) economic factors (annual discount and inflation rates), and (iv) desired number of maintenance actions over the life-cycle. The output of the procedure is a set of maintenance scenarios obtained by applying multi-objective optimization considering probabilistic cost model and a component-based and sub-system-based combinatorial analysis model to give multiple options to the decision-maker. These options contain performance metric and maintenance cost for each to-berepaired/replace component and corresponding time of actions and type of action. The outcome of the developed system leads to optimal decision-making regarding repair and replacement of the structure during the life-cycle in terms of time and type of action with respect to system requirements, structure specifications, and available budget.

\section{References}

[1] Frangopol, D. M. (2002). Reliability deterioration and lifetime maintenance cost optimization. The first international ASRANet colloquium on integrating structural reliability analysis with advanced structural analysis. Citeseer.

[2] Barone, G., \& Frangopol, D. (2014). Life-cycle maintenance of deteriorating structures by multi-objective optimization involving reliability, risk, availability, hazard and cost. Structural Safety , 48, 40-50. https://doi.org/10.1016/j.strusafe.2014.02.002

[3] Barone, G., Frangopol, D. M., \& Soliman, M. (2013). Optimization of life-cycle maintenance of deteriorating bridges with respect to expected annual system failure rate and expected cumulative cost. Journal of Structural Engineering , 140 (2), 04013043. https://doi.org/10.1061/(ASCE)ST.1943-541X.0000812

[4] Saydam, D., \& Frangopol, D. M. (2014). Risk-based maintenance optimization of deteriorating bridges. American Society of Civil Engineers, 141 (4), 04014120. https://doi.org/10.1061/(ASCE)ST.1943-541X.0001038

[5] Akgul, F. (2002). Lifetime system reliability prediction for multiple structure types in a bridge network. PhD Dissertation, University of Colorado.

[6] Saydam, D. (2013). Reliability and risk of structural systems under progressive and sudden damage. PhD Dissertation, Lehigh University.

[7] Rausand, M., \& Arnljot, H. Ã. (2004). System reliability theory: models, statistical methods, and applications (Vol. 396). John Wiley \& Sons.

[8] Petcherdchoo, A., Neves, L. A., \& Frangopol, D. M. (2008). Optimizing lifetime condition and reliability of deteriorating structures with emphasis on bridges. Journal of Structural Engineering, 134 (4), 544-552. https://doi.org/10.1061/(ASCE)0733-9445(2008)134:4(544)

[9] Montazeri, N. (2018). Multi-objective optimization for life-cycle reliability-based bridge maintenance scheduling. PhD Dissertation, Northeastern University.

[10] Legriel, J., Le Guernic, C., Cotton, S., \& Maler, O. (2010). Approximating the Pareto Front of Multi-criteria Optimization Problems. In TACAS (pp. 69-83). Berlin, Heidelberg: Springer. https://doi.org/10.1007/978-3-642-12002-2_6

[11] Certa, A., Galante, G., Lupo, T., \& Passannanti, G. (2011). Determination of Pareto frontier in multi-objective maintenance optimization. Reliability Engineering and System Safety , 96 (7), 861-867. http://dx.doi.org/10.1016/j.ress.2010.12.019 